\title{
Erratum to: Ultrafast nuclear myocardial perfusion imaging on a new gamma camera with semiconductor detector technique: first clinical validation
}

Ronny R. Buechel • Bernhard A. Herzog • Lars Husmann • Irene A. Burger • Aju P. Pazhenkottil • Valerie Treyer • Ines Valenta • Patrick von Schulthess • René Nkoulou • Christophe A. Wyss • Phillip A. Kaufmann

Published online: 3 April 2011

(C) Springer-Verlag 2011

Erratum to: Eur J Nucl Med Mol Imaging

DOI 10.1007/s00259-009-1375-7

Table 2 contained erroneous data. The corrected table is reproduced here.

Table 2 Diagnostic performance of CZT compared to conventional SPECT

TP true positive, FP false positive, $T N$ true negative, $F N$ false negative, Sens sensitivity, Spec specificity, $P P V$ positive predictive value, $N P V$ negative predictive value

\begin{tabular}{lllllllllll}
\hline & $\mathrm{n}$ & TP & FP & TN & FN & Sens & Spec & PPV & NPV & Accuracy \\
\hline Per patient & 75 & 48 & 0 & 24 & 3 & $94 \%$ & $100 \%$ & $100 \%$ & $89 \%$ & $96 \%$ \\
Per territory & & & & & & & & & & \\
All vessels & 225 & 65 & 4 & 152 & 4 & $94 \%$ & $97 \%$ & $94 \%$ & $97 \%$ & $96 \%$ \\
LAD & 75 & 12 & 2 & 58 & 3 & $80 \%$ & $97 \%$ & $86 \%$ & $95 \%$ & $93 \%$ \\
LCX & 75 & 7 & 1 & 66 & 1 & $88 \%$ & $99 \%$ & $88 \%$ & $99 \%$ & $97 \%$ \\
RCA & 75 & 46 & 1 & 28 & 0 & $100 \%$ & $97 \%$ & $98 \%$ & $100 \%$ & $99 \%$ \\
\hline
\end{tabular}

The online version of the original article can be found at http://dx.doi. org/10.1007/s00259-009-1375-7.

R. R. Buechel • B. A. Herzog • L. Husmann · I. A. Burger •

A. P. Pazhenkottil $\cdot$ V. Treyer $\cdot$ I. Valenta $\cdot$ P. von Schulthess $\cdot$

R. Nkoulou $\cdot$ C. A. Wyss $\cdot$ P. A. Kaufmann $(\bowtie)$

Cardiac Imaging, University Hospital Zurich,

Ramistrasse 100,

Zurich CH-8091, Switzerland

e-mail:pak@usz.ch

P. A. Kaufmann

Zurich Center for Integrative Human Physiology (ZIHP),

University Zurich,

Zurich, Switzerland 